\author{
V. Mericq $\cdot$ K. K. Ong $\cdot$ R. Bazaes $\cdot$ V. Peña $\cdot$ A. Avila $\cdot$ \\ T. Salazar · N. Soto $\cdot$ G. Iñiguez $\cdot$ D. B. Dunger
}

\title{
Longitudinal changes in insulin sensitivity and secretion from birth to age three years in small- and appropriate-for-gestational-age children
}

Received: 2 June 2005 / Accepted: 12 September 2005 / Published online: 8 November 2005

(C) Springer-Verlag 2005

\begin{abstract}
Aims/hypothesis: Insulin resistance and type 2 diabetes risk in human subjects who were small-for-gestationalage (SGA) at birth may be a consequence of rapid early postnatal weight gain. Materials and methods: We prospectively studied early changes in fasting insulin sensitivity and insulin secretion, assessed by a short intravenous glucose tolerance test that was conducted several times from birth to 3 years of age in 55 SGA (birthweight below fifth percentile) newborns and in 13 newborns with a birthweight appropriate for gestational age (AGA). Results: Most SGA infants showed postnatal upward weight centile crossing and by 3 years were similar in size to AGA infants. SGA infants had lower pre-feed insulin levels at postnatal age $48 \mathrm{~h}$ than AGA infants (median 34.4 vs $59.7 \mathrm{pmol} / 1$, $p<0.05$ ), but by the age of 3 years they had higher fasting insulin levels (median 38.9 vs $23.8 \mathrm{pmol} / \mathrm{l}, p<0.005$ ), which were related to rate of weight gain between 0 and 3 years $(r=0.47, p=0.0003)$. First-phase insulin secretion did not differ between SGA and AGA infants, but SGA infants had a lower glucose disposition index (beta cell compensation) (median 235 vs $501 \mathrm{~min} \mathrm{mmol}^{-1} \mathrm{l}^{-1}, p=0.02$ ), which persisted after allowing for postnatal weight gain $(p=0.009)$. Conclusions/interpretation: SGA infants showed a marked
\end{abstract}

V. Mericq $\cdot$ R. Bazaes $\cdot$ V. Peña $\cdot$ A. Avila $\cdot$ T. Salazar $\cdot$ N. Soto $\cdot$ G. Iñiguez

Institute of Maternal and Child Research, University of Chile, Santiago, Chile

K. K. Ong

Medical Research Council Epidemiology Unit,

Cambridge, UK

K. K. Ong · D. B. Dunger

Department of Paediatrics, University of Cambridge,

Cambridge, UK

V. Mericq $(\bowtie)$

Instituto de Investigaciones Materno Infantil,

Casilla 226-3,

Santiago, Chile

e-mail: vmericq@med.uchile.cl

Tel.: +56-2-4248280

Fax: +56-2-4247240 transition from lower pre-feed insulin and increased insulin sensitivity at birth to insulin resistance over the first 3 years of life. This transition was related to rapid postnatal weight gain, which could indicate a propensity to central fat deposition. The additional observation of reduced compensatory beta cell secretion underlines the need for long-term surveillance of glucose homeostasis in all SGA subjects, whether or not they show postnatal catch-up growth.

Keywords Birthweight - Catch-up growth · Disposition index · Human · Insulin secretion · Insulin sensitivity

Abbreviations AGA: appropriate for gestational age $\mathrm{AIR}_{\mathrm{g}}$ : incremental insulin area under for glucose 0 $10 \mathrm{~min}$ - ALSPAC: Avon Longitudinal Study of Pregnancy and Childhood - HOMA-IR: homeostasis model assessment of insulin resistance - SGA: small for gestational age

\section{Introduction}

Birth size that is small for gestational age (SGA) is associated with short adult stature [1] and increased risk of type 2 diabetes [2], cardiovascular disease [3] and insulin resistance [4]. Recent data indicate that most of these consequences are also related to rapid postnatal patterns of weight gain $[5,6]$. Body fat mass and fat distribution are important determinants of insulin sensitivity, and associations between smaller size at birth and increased risks of insulin resistance are often evident only after allowing for current adiposity [7].

In young adults insulin sensitivity is decreased in SGA subjects compared with subjects whose birth size was appropriate for gestational age (AGA), in spite of a similar BMI [4]. Hence, it has been suggested that a combination of both pre- and postnatal factors may determine insulin sensitivity in SGA infants. Most SGA infants display postnatal upward weight centile crossing or catch-up growth [1]. In some populations this may lead to higher childhood weight and central fat distribution [8]. 
We recently reported that insulin sensitivity and secretion in 1-year-old SGA infants were closely related to patterns of postnatal weight gain. At age 1, increased fasting insulin levels, an indicator of insulin resistance, were associated with greater postnatal weight gain and larger current BMI, whereas insulin secretion was more closely related to early gains in length [9]. We have continued to follow this birth cohort up to the third year of life in order to further explore the early development of insulin resistance and insulin secretion, as markers of future risk of type 2 diabetes.

\section{Subjects and methods}

Study protocol

During the first year of life, 85 SGA and 23 AGA children attended the clinic for anthropometric evaluation and a short IVGTT; these numbers decreased to 66 SGA and 20 AGA during the second year and to 55 SGA and 13 AGA during the third year of the evaluation. Data on body size and insulin levels at birth and at age 1 year did not differ significantly between subjects who completed the 3-year follow-up and those who did not attend at 3 years.

Fifty-five SGA infants, defined as having a birthweight below the fifth percentile (corresponding to $-1.65 \mathrm{SD}$ ) for gestational age using the Chilean birthweight reference [10], and 13 AGA infants (birthweight above the fifth percentile) completed participation in the study. Subjects were recruited at birth from the neonatal units of Hospital San Borja Arriarán and Hospital Sótero del Río, and subsequently completed follow-up to the third year of life at the Pediatric Endocrine Unit, Institute of Maternal and Child Research, University of Chile. All infants had a gestational age between 37 and 41 weeks and during the second day of life underwent a screening clinical evaluation to exclude those with significant medical, neurological or genetic conditions. All infants were exclusively breast-fed for a mean of 3.7 months; there were no differences between those born SGA or AGA. Thereafter, they received standard formula and solid meals as recommended by the American Academy of Pediatrics. No infants were taking any medication that could interfere with growth or appetite. A complete record of parental, pregnancy and perinatal data was completed at entry.

The study protocol was approved by the San Borja Arriarán Hospital Institutional Review Board. All parents or guardians of the participants gave written informed consent.

\section{Measurements}

All children had weight and length measured at birth, and at ages 1, 2 and 3 years by one nurse (A. Avila). Supine length was measured using a firm box with a fixed headboard and a movable footboard with the feet placed per-
Table 1 Anthropometric and hormonal data in small-forgestational-age (SGA) and appropriate-for-gestational-age (AGA) infants at birth and 1 and 3 years

Data are mean $\pm \mathrm{SE}$ or median (interquartile range)

${ }^{\mathrm{a}} p<0.01,{ }^{\mathrm{b}} p<0.05$, SGA vs AGA SDS, SD score

\begin{tabular}{|c|c|c|}
\hline & SGA $(n=55)$ & AGA $(n=13)$ \\
\hline Weight at birth (SDS) & $-2.07 \pm 0.08^{\mathrm{a}}$ & $0.76 \pm 0.30$ \\
\hline Weight at 1 year (SDS) & $-0.72 \pm 0.14^{\mathrm{a}}$ & $0.20 \pm 0.31$ \\
\hline Weight at 3 years (SDS) & $-0.03 \pm 0.17$ & $0.39 \pm 0.30$ \\
\hline Change in weight from 1 to 3 years (SDS) & $0.71 \pm 0.13^{\mathrm{b}}$ & $0.20 \pm 0.14$ \\
\hline Length at birth (SDS) & $-1.64 \pm 0.12^{\mathrm{a}}$ & $0.15 \pm 0.21$ \\
\hline Length at 1 year (SDS) & $-0.84 \pm 0.11^{\mathrm{b}}$ & $0.33 \pm 0.30$ \\
\hline Length at 3 years (SDS) & $-0.60 \pm 0.13$ & $-0.35 \pm 0.25$ \\
\hline Change in length from 1 to 3 years (SDS) & $0.26 \pm 0.09$ & $-0.09 \pm 0.21$ \\
\hline Ponderal index at birth & $24.7 \pm 0.2^{\mathrm{a}}$ & $27.8 \pm 0.5$ \\
\hline BMI at 1 year $\left(\mathrm{kg} / \mathrm{m}^{2}\right)$ & $17.0 \pm 0.2^{\mathrm{b}}$ & $18.1 \pm 0.4$ \\
\hline BMI at 3 years $\left(\mathrm{kg} / \mathrm{m}^{2}\right)$ & $16.2 \pm 0.2$ & $16.7 \pm 0.3$ \\
\hline Fasting insulin at $48 \mathrm{~h}(\mathrm{pmol} / \mathrm{l})$ & $34.4(21.9-51.4)^{\mathrm{b}}$ & $59.7(37.5-84.4)$ \\
\hline Fasting insulin at 1 year (pmol/1) & $19.8(11.8-29.0)$ & $19.1(10.5-27.7)$ \\
\hline Fasting insulin at 3 years $(\mathrm{pmol} / \mathrm{l})$ & $38.9(31.3-46.8)^{\mathrm{a}}$ & $23.8(14.2-41.2)$ \\
\hline HOMA-IR at $48 \mathrm{~h}([\mathrm{pmol} / 1] \times[\mathrm{mmol} / 1])$ & $5.2(3.1-8.3)^{\mathrm{b}}$ & $9.6(5.6-11.3)$ \\
\hline HOMA-IR at 1 year $([\mathrm{pmol} / 1] \times[\mathrm{mmol} / 1])$ & $4.2(2.4-7.1)$ & $3.8(2.2-6.5)$ \\
\hline HOMA-IR at 3 years $([\mathrm{pmol} / 1] \times[\mathrm{mmol} / 1])$ & $8.5(6.5-11.0)^{\mathrm{a}}$ & $4.9(2.8-8.8)$ \\
\hline Glucose at $48 \mathrm{~h}(\mathrm{mmol} / \mathrm{l})$ & $3.4(2.8-4.0)^{\mathrm{a}}$ & $3.8(3.5-4.3)$ \\
\hline Fasting glucose at 1 year $(\mathrm{mmol} / \mathrm{l})$ & $4.8(4.5-5.1)$ & $4.7(4.5-5.5)$ \\
\hline Fasting glucose at 3 years $(\mathrm{mmol} / \mathrm{l})$ & $4.8(4.6-5.0)$ & $4.4(4.3-5.0)$ \\
\hline Insulin secretion at 1 year $\left(\left[\mathrm{pmol} \mathrm{l}^{-1}\right] \times \min \right)$ & $1571(682-2386)$ & $883(630-2769)$ \\
\hline Insulin secretion at 3 years $\left(\left[\mathrm{pmol} \mathrm{1} 1^{-1}\right] \times \min \right)$ & 2062 (1494-2847) & $1496(850-3243)$ \\
\hline Disposition index at 1 year $\left(\left[\min \mathrm{mmol}^{-1}\right] \times 1\right)$ & $377(176-548)$ & $238(190-743)$ \\
\hline Disposition index at 3 years $\left(\left[\min \mathrm{mmol}^{-1}\right] \times 1\right)$ & $235(153-311)^{\mathrm{a}}$ & $501(256-683)$ \\
\hline
\end{tabular}


pendicular to the plane determined by the supine length of the infant. Weight was measured using a manual scale with a 10-g graduation (Seca, Quickmedical, Snoqualme, WA, USA).

Forty-eight hours after birth, a pre-feeding 3-ml blood sample was obtained for determination of glucose, insulin and other markers of insulin sensitivity and secretion, as previously reported [11]. At 1 and 3 years of age, a short IVGTT was performed after an overnight fast (mean duration of fast, $9 \mathrm{~h}$ ). Two venous access lines were established in contralateral antecubital veins. Glucose $(25 \%$ dextrose solution) was administered at a dose of $0.5 \mathrm{~g} / \mathrm{kg}$ (maximum $35 \mathrm{~g}$ ) by continuous infusion over $3 \mathrm{~min}$, according to a protocol recommended by National Diabetes Data Group for a short IVGTT. Blood samples were obtained at $-5,0,1,3,5$ and $10 \mathrm{~min}$ (where 0 is the start of glucose infusion) for determination of glucose and insulin levels. Glucose was measured immediately, whereas samples for insulin were kept on ice and centrifuged within $30 \mathrm{~min}$, and sera were frozen at $-20^{\circ} \mathrm{C}$.

\section{Assays}

Blood glucose concentration was determined using a commercial glucometer (Accutrend Sensor Comfort; Roche Diagnostics. Basel, Switzerland), which yields values $8 \pm 5 \%$ $($ mean \pm SD) higher than standard enzymatic methods with a correlation coefficient of 0.987 for glycaemia between 2.2 and $8.0 \mathrm{mmol} / \mathrm{l}$.

Serum insulin was measured using a commercial radioimmunoassay from Diagnostic System Laboratories (Webster, TX, USA). This assay has a cross-reactivity of $27.5 \%$ with proinsulin and $25 \%$ with insulin-32, 33. The sensitivity of this assay was $5.6 \mathrm{pmol} / \mathrm{l}$. Intra-assay and interassay coefficients of variation were 3.8 and $4.7 \%$, respectively.

Calculations and analysis

Weights and lengths at birth and at ages 1 and 3 years were converted to SD scores in order to adjust for chronological age and sex using the National Center for Health Statistics (NCHS) growth reference, which has been shown to be applicable to the Chilean population [12]. BMI was calculated at age 1 and 3 years (weight/length ${ }^{2}$ ).

Insulin resistance was estimated using the homeostasis model (HOMA-IR) using the formula (insulin [pmol/ 1] $\times$ glucose [mmol/1]/22.5) [13]. HOMA-IR values showed extremely high correlation with fasting insulin levels $(r=0.96, p<0.0001)$. Results of analyses using HOMA-IR were therefore nearly identical to those using fasting insulin levels. First-phase insulin secretion during the short IVGTT was expressed as the incremental insulin area under the curve for glucose $0-10 \mathrm{~min}\left(\mathrm{AIR}_{\mathrm{g}}\right)$, calculated using the trapezoidal rule $\left(\left[\mathrm{pmol} \mathrm{l}^{-1}\right] \times \mathrm{min}\right)$. In order to account for the modulating effect of insulin sensitivity on the beta cell, the glucose disposition index was calculated as (insulin secretion $\left.\left[\mathrm{AIR}_{\mathrm{g}}\right] / \mathrm{HOMA}-\mathrm{IR}\right)\left(\left[\mathrm{min} \mathrm{mmol}^{-1}\right] \times 1\right)[14]$.
Results are expressed as mean \pm SEM. Differences between groups were assessed by ANOVA for normally distributed variables, and by non-parametric tests (Mann-Whitney $U$ test or Kruskall-Wallis test) for non-normally distributed variables (fasting insulin, and leptin). In order to examine the relationships between continuous variables, non-normally distributed variables were transformed to normal distributions by calculating logarithms. Univariate correlations were performed and Pearson's correlation coefficients $(r)$ are


Age at assessment

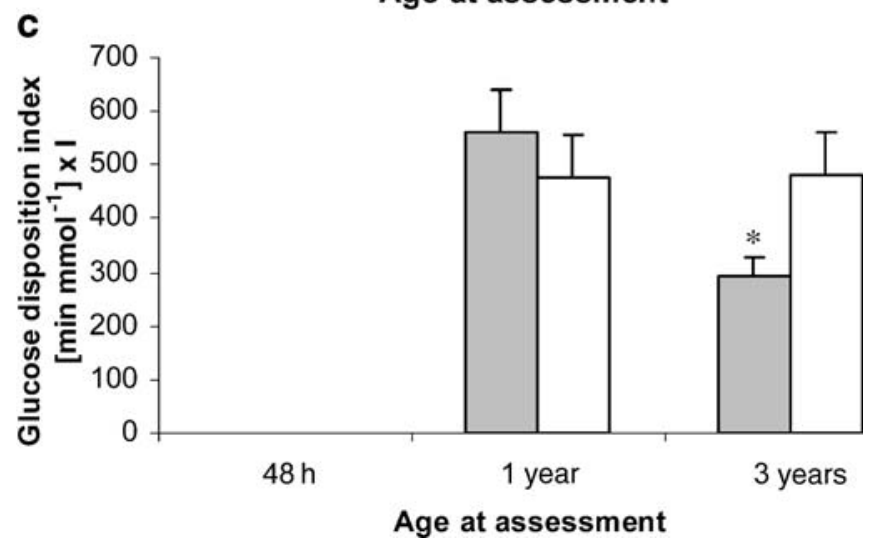

Fig. 1 Longitudinal measures of (a) weight SD score (SDS), (b) fasting insulin levels and (c) glucose disposition index from birth to 3 years in small-for-gestational age (SGA; $n=55$; shaded bars) and appropriate-for-gestational-age (AGA; $n=13$; open bars) infants. Means $\pm 95 \%$ CI are shown for weight SD scores, and geometric means $+95 \%$ CI for fasting insulin and glucose disposition index. ${ }^{*} p<0.05$ for SGA vs AGA. At the age of 1 year, fasting insulin levels were only higher in SGA infants who showed weight catch-up compared with other infants $(p<0.05$ [9]) 
displayed. All statistics were run on SPSS 10.0 for Windows, and $p<0.05$ was considered to be significant.

\section{Results}

Compared with the AGA controls, SGA infants were significantly lighter and shorter at birth, and they continued to show significant gains in weight SD score throughout the 3 years of follow-up (Table 1, Fig. 1). In contrast to the time of birth and at 1 year of age, SGA infants were no longer significantly smaller than AGA infants by the age of 3 years (Table 1).

As previously reported for the complete cohort [11], at age $48 \mathrm{~h}$ the SGA infants had lower insulin levels (median 34.4 vs $59.7 \mathrm{pmol} / \mathrm{l}, p=0.03$; Table 1) than the AGA infants. In contrast, at age 3 years, the SGA infants had higher fasting insulin levels (median 38.9 vs 23.8 pmol/l, $p=0.005$; Fig. 1b) compared with AGA infants. Similarly, SGA infants had higher HOMA-IR levels at 3 years (median 8.5 vs $4.9 \mathrm{pmol} /$ $1 \times \mathrm{mmol} / 1 ; p=0.007$; Table 1 ), and this was independent of current body weight $(p=0.009)$ or BMI $(p=0.01)$. In a multiple regression analysis, the strongest determinant of fasting insulin at age 3 years was the rate of weight gain between birth and 3 years $(r=0.47, p=0.0003$; Fig. 2$)$, with no further significant contribution of birthweight or current BMI.

First-phase insulin secretion $\left(\mathrm{AIR}_{\mathrm{g}}\right)$ at age 3 years did not differ between SGA and AGA infants (Table 1), and was positively related to postnatal weight gain from birth to 3 years $(r=0.42, p=0.003)$ and to fasting insulin level $(r=0.45$, $p=0.002$ ). The glucose disposition index, which accounts for the modulating effect of insulin sensitivity on the beta cell, was lower in SGA infants at age 3 years than in AGA infants (median 235 vs $501\left[\mathrm{~min} \mathrm{mmol}^{-1}\right] \times 1, p=0.02$; Table 1). In a multiple regression model, the glucose disposition index was
Table 2 Multiple regression model showing the of determinants of the glucose disposition index (a marker of beta cell compensatory insulin secretion) at age 3 years in SGA and AGA children

\begin{tabular}{lcc}
\hline Variable & Regression coefficient & $p$-value \\
\hline Birthweight & 0.36 & $p=0.009$ \\
Postnatal weight gain & 0.31 & $p=0.03$ \\
Sex & -0.12 & $p=0.34$ \\
\hline
\end{tabular}

positively related to birthweight $(p=0.009)$ and rate of postnatal weight gain ( $p=0.03$; Table 2$)$.

\section{Discussion}

This study presents unique longitudinal data on the development of insulin resistance and insulin secretion in term SGA and AGA infants from birth to age 3 years. We have previously reported that SGA infants have lower insulin levels at around the time of birth than AGA infants [11], but by the age of 1 year SGA infants, who showed catch-up weight gain (gain in weight SD score, $>0.67$ ), had higher fasting insulin levels and insulin resistance [9]. We now show that gains in weight SD score, or upward weight centile crossing, continued to the age of 3 years in SGA infants, and their insulin resistance also continued to progress during this period. By age 3 there were no differences in weight or BMI between the two groups and insulin resistance in SGA infants was related to rapid weight gain during infancy.

In many population studies, reduced fetal growth has been reported to be a risk factor for insulin resistance and type 2 diabetes [2, 4, 9, 15-17]. In particular, subjects who were small or thin at birth but subsequently developed obesity in childhood or in adulthood have the highest risk of developing insulin resistance [9, 15-17]. Smaller infants
Fig. 2 Fasting insulin resistance (HOMA IR, [pmol/1] $\times$ $[\mathrm{mmol} / 1])$ at age 3 years related to rate of weight gain between 0 and 3 years in infants with normal birthweight (AGA, filled-squares) and low birthweight (SGA, open symbols). Regression for total population: $r=0.47, p=0.0003$ SDS, SD score

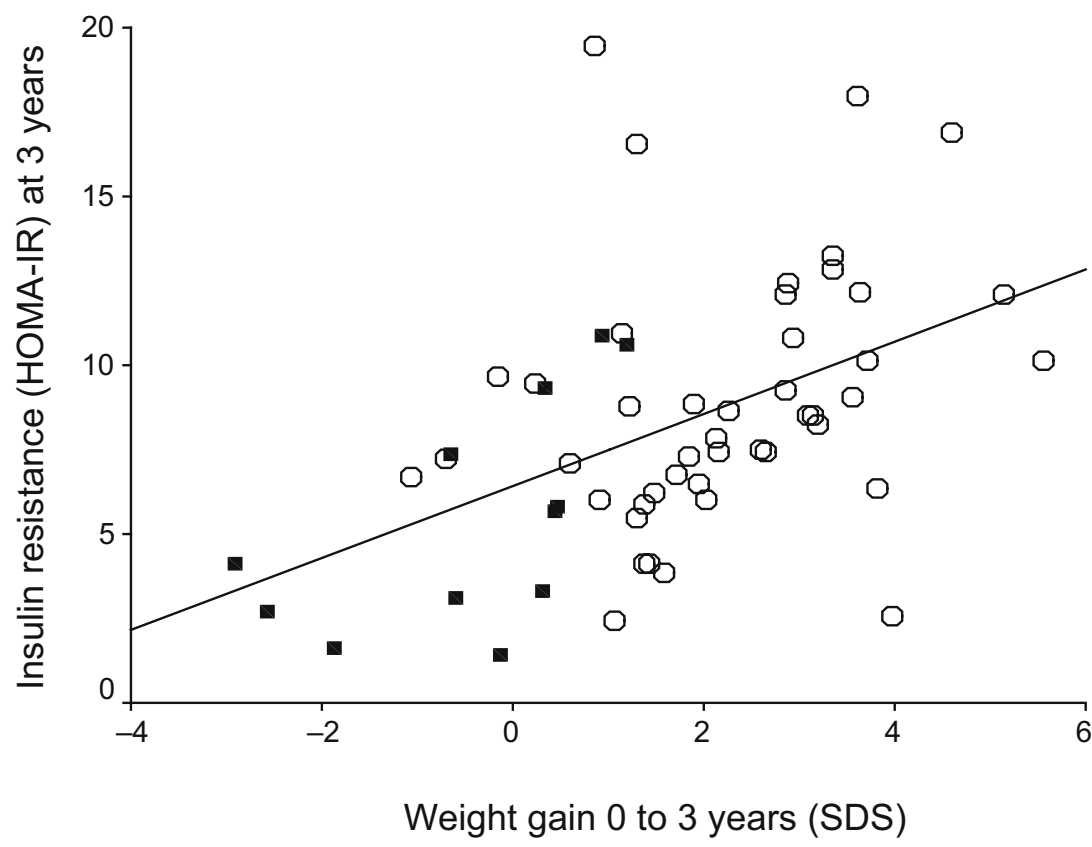


at birth are more likely to show faster postnatal weight gain. Such rapid catch-up growth may be initiated as a compensatory mechanism following in utero growth restraint; however, longer-term follow-up indicates that such early growth patterns are predictive of childhood and later obesity [8, 18].

In our study, the majority of SGA infants (48/55, 87\%) showed postnatal catch-up weight gain between birth and age 3 years (as defined by gain in weight SD score $>0.67$ ), and rate of weight gain during this period was the most important predictor of insulin resistance at age 3 years. Rapid early weight gain could influence a more central or visceral distribution of body fat, rather than subcutaneous fat. Many studies have shown that fat distribution influences metabolism independently of the effects of total body fat stores [19, 20]. In the Avon Longitudinal Study of Pregnancy and Childhood (ALSPAC) cohort, low birthweight followed by rapid infancy weight gain was associated with increased risk of obesity and larger waist circumference [8]. Similar findings have been observed in US cohorts [21] and recently in a Swedish longitudinal study [22].

Studies to assess the contribution of visceral fat to insulin resistance have shown that the omental adipose bed is a depot that drains directly into the portal vein, and is particularly associated with insulin resistance, probably as a result of the effects of increased non-esterified fatty acid flux to the liver [23]. Recent evidence suggests that not only non-esterified fatty acids but other adipokines are directly or indirectly involved in this process, and these may be dysregulated in SGA adipose tissue [24]. In this prospective cohort study, we recently showed that levels of the adipokine adiponectin declined from the first to the second year of life, and the rate of fall was related to age and greater weight gain in SGA infants [25]. In that analysis, adiponectin levels were unrelated to the degree of insulin resistance. However, in addition to adiponectin's effects on promoting insulin sensitivity, insulin may stimulate adiponectin secretion, and the resulting cross-sectional associations between insulin and adiponectin levels may also be influenced by body fat and fat distribution [26]. Greater understanding of adipokine regulation could inform future treatment strategies to control disease states associated with increased adiposity, an important outcome in view of the growing worldwide epidemic of obesity [20, 27].

The relationship between insulin sensitivity and compensatory insulin secretion is critical to the risk of the development of glucose intolerance [14]. In our study, SGA infants at age 3 years had a reduced disposition index, which may indicate an early deficiency in their compensatory first-phase insulin response. In a separate study of 850 children from the ALSPAC cohort aged 8 years, we showed that whereas the rate of early postnatal weight gain was an important determinant of obesity and insulin resistance, compensatory insulin secretion for the degree of insulin resistance was related to height gain and IGF-I levels [7]. In the present cohort, we reported earlier that insulin secretion at age 1 year was related to height gain [9], and it is possible that insulin secretion in response to growth-hormone-related insulin resistance could promote catch-up growth, particularly in length [28]. However, we could not confirm those associations with insulin secretion at age 3 years, perhaps because of the reduced sample size.

In conclusion, the development of insulin resistance in children born SGA occurs during early postnatal life, concurrently with their rapid early postnatal catch-up weight gain. It is speculated from recent studies that these associations may be linked to increased central fat deposition. By the age of 3 years the relationship between insulin sensitivity and insulin secretion may be perturbed in some of these SGA infants, as reflected in a reduced glucose disposition index. These findings have important implications for the reported association between size at birth, early weight gain and the risk of type 2 diabetes.

Acknowledgements This study was supported by Fondo Nacional de Desarrollo Cientifico y Techologico (National Fund for Scientific and Technological Research; Fondecyt; grant no.: N 1000939). D. B. Dunger is supported by the Wellcome Trust and the Juvenile Diabetes Research Foundation.

\section{References}

1. Hokken-Koelega AC, De Ridder MA, Lemmen RJ et al (1995) Children born small for gestational age: do they catch up? Pediatr Res 38:267-271

2. Hales CN, Barker DJ, Clark PM et al (1991) Fetal and infant growth and impaired glucose tolerance at age 64. BMJ 303: 1019-1022

3. Barker DJ, Osmond C, Golding J, Kuh D, Wadsworth ME (1989) Growth in utero, blood pressure in childhood and adult life, and mortality from cardiovascular disease. BMJ 298:564 567

4. Jaquet D, Gaboriau A, Czernichow P, Levy-Marchal C (2000) Insulin resistance early in adulthood in subjects born with intrauterine growth retardation. J Clin Endocrinol Metab 85: 1401-1406

5. Eriksson JG, Forsen T, Tuomilehto J, Winter PD, Osmond C, Barker DJ (1999) Catch-up growth in childhood and death from coronary heart disease: longitudinal study. BMJ 318:427-431

6. Eriksson JG, Forsen T, Tuomilehto J, Jaddoe VW, Osmond C, Barker DJ (2002) Effects of size at birth and childhood growth on the insulin resistance syndrome in elderly individuals. Diabetologia 45:342-348

7. Ong KK, Petry CJ, Emmett PM et al (2004) Insulin sensitivity and secretion in normal children related to size at birth, postnatal growth, and plasma insulin-like growth factor-I levels. Diabetologia 47:1064-1070

8. Ong KK, Ahmed ML, Emmett PM, Preece MA, Dunger DB (2000) Association between postnatal catch-up growth and obesity in childhood: prospective cohort study. BMJ 320:967971

9. Soto N, Bazaes RA, Pena V et al (2003) Insulin sensitivity and secretion are related to catch-up growth in small-for-gestational-age infants at age 1 year: results from a prospective cohort. J Clin Endocrinol Metab 88:3645-3650

10. Juez G (1989) Intrauterine growth curve for the appropriate diagnosis of intrauterine growth retardation. Rev Med Chile 117:1311

11. Bazaes RA, Salazar TE, Pittaluga E et al (2003) Glucose and lipid metabolism in small for gestational age infants at 48 hours of age. Pediatrics 111:804-809 
12. Youlton R, Valenzuela C (1990) Growth patterns in height and weight in children aged 0 to 17 years and cranial circumference in children aged 0 to 2 years from medium-high and high socioeconomic status in Santiago. Comparison with growth in children from medium-low and low status in the Northern area of Santiago. Rev Chil Pediatr Spec:1-22

13. Gungor N, Saad R, Janosky J, Arslanian S (2004) Validation of surrogate estimates of insulin sensitivity and insulin secretion in children and adolescents. J Pediatr 144:47-55

14. Bergman RN, Finegood DT, Kahn SE (2002) The evolution of beta-cell dysfunction and insulin resistance in type 2 diabetes. Eur J Clin Investig 32 (Suppl 3):35-45

15. Bavdekar A, Yajnik CS, Fall CH et al (1999) Insulin resistance syndrome in 8-year-old Indian children: small at birth, big at 8 years, or both? Diabetes 48:2422-2429

16. Whincup PH, Cook DG, Adshead F et al (1997) Childhood size is more strongly related than size at birth to glucose and insulin levels in 10-11-year-old children. Diabetologia 40:319-326

17. Yarbrough DE, Barrett-Connor E, Kritz-Silverstein D, Wingard DL (1998) Birth weight, adult weight, and girth as predictors of the metabolic syndrome in postmenopausal women: the Rancho Bernardo study. Diabetes Care 21:1652-1658

18. Stettler N, Stallings VA (2002) Adult obesity and growth in childhood. Association of birth weight with adult weight is confounded by maternal body mass index. BMJ 324:674

19. Reaven GM (1995) The fourth musketeer-from Alexandre Dumas to Claude Bernard. Diabetologia 38:3-13
20. Lyon CJ, Law RE, Hsueh WA (2003) Minireview: adiposity, inflammation, and atherogenesis. Endocrinology 144:21952200

21. Stettler N, Zemel BS, Kumanyika S, Stallings VA (2002) Infant weight gain and childhood overweight status in a multicenter cohort study. Pediatrics 109:194-199

22. Ekelund U, Ong K, Linne Y et al (2004) Both infancy and childhood weight gain predict obesity risk at age 17 years: prospective birth cohort study (SWEDES). Obes Res 12:A186

23. Mittelman SD, Van Citters GW, Kirkman EL, Bergman RN (2002) Extreme insulin resistance of the central adipose depot in vivo. Diabetes 51:755-761

24. Jaquet D, Gaboriau A, Czernichow P, Levy-Marchal C (2001) Relatively low serum leptin levels in adults born with intrauterine growth retardation. Int J Obes Relat Metab Disord 25: $491-495$

25. Iniguez G, Soto N, Avila A et al (2004) Adiponectin levels in the first two years of life in a prospective cohort: relations with weight gain, leptin levels and insulin sensitivity. J Clin Endocrinol Metab 89:5500-5503

26. Dunger D, Ong K (2004) Abundance of adiponectin in the newborn. Clin Endocrinol (Oxf) 61:416-417

27. Matsuzawa Y, Shimomura I, Kihara S, Funahashi T (2003) Importance of adipocytokines in obesity-related diseases. Horm Res 60 (Suppl 3):56-59

28. Woods KA, van Helvoirt M, Ong KK et al (2002) The somatotropic axis in short children born small for gestational age: relation to insulin resistance. Pediatr Res 51:76-80 